Voix et Images

volxetimages

\title{
Discours épistolaire et paroles en jeu
}

\section{Bernard Andrès}

Volume 7, numéro 1, automne 1981

\section{Adrien Thério}

URI : https://id.erudit.org/iderudit/200315ar

DOI : https://doi.org/10.7202/200315ar

Aller au sommaire du numéro

Éditeur(s)

Les Presses de l'Université du Québec

ISSN

0318-9201 (imprimé)

1705-933X (numérique)

Découvrir la revue

Citer cet article

Andrès, B. (1981). Discours épistolaire et paroles en jeu. Voix et Images, 7(1), 191-193. https://doi.org/10.7202/200315ar d'utilisation que vous pouvez consulter en ligne.

https://apropos.erudit.org/fr/usagers/politique-dutilisation/ 


\section{Théâtre \\ Discours épistolaire et paroles en jeu}

par Bernard Andrés

Plutôt que de revenir sur des spectacles déjà fort bien couverts ailleurs, dans des chroniques ponctuelles auxquelles je renvoie le lecteur, je préfère ici m'arrêter sur des impressions d'ensemble touchant à l'année écoulée; particulièrement certaines pièces moins choyées par la critique et dont le souvenir encore vif $\mathrm{m}^{\prime}$ assurent de l'impact qu'elles purent avoir. Quelques traces, donc, de la saison passée, exception faite des mois d'absence au tournant des deux années, où je n'ai pu suivre que de loin la production théâtrale montréalaise (là-bas, c'était la tournée française de Louisette Dussault, c'était aussi Barbeau revu et corrigé par une troupe parisienne, qui s'escrimait à vouloir faire passer les vessies d'Une brosse pour les lanternes d'un Ivre pour vivre argotique et cahoteux).

Ne dire qu'un mot, donc, de l'excellente mise en scène de Galilée à La Grande Réplique ', du beau travail textuel et scénique de Jocelyne Beaulieu et Michelle Rossignol au Théâtre d'aujourd'hui : J'ai beaucoup changé depuis ${ }^{2}$, de la reprise de Moman de Louisette Dussault, en tournée québécoise à l'automne, puis à la Comédie nationale au printemps, à l'occasion de la publication du texte chez Boréal Express ${ }^{3}$. Passer discrètement sur Cristal, de l'Eskabel et sur La St-Jean du $\rho^{\prime} t i t$ monde, de G. Turp, au Théâtre d'aujourd'hui, mais insister davantage sur J'te l'parle mieux quand j'te l'écris. au même théâtre (février-avril 81).

Comme on le sait, cette pièce est tirée du recueil de lettres québécoises, Chers nous autres, de Robert Blondin 4 . Elle a été conçue par Suzanne Aubry et mise en scène par Jacques Rossi. II s'agit d'un montage de lettres échangées par des citoyen(ne)s "ordinaires" pendant des décennies de petite histoire du Québec. Aventures et mésaventures amoureuses, soucis quotidiens, anecdotes familiales, tissent un réseau d'informations, parallèlement à I'Histoire des "hauts faits" canadiens dont se repaissent les savants manuels. On connaît assez l'esprit du Boréal Express pour savoir à partir de quel matériau et dans quelle optique de réappropriation culturelle s'élabora le recueil de Blondin (en collaboration avec Gilles Lamontagne et Marie-Andrée Vinet). 
Des quelques douze mille lettres rapaillées à la grandeur du pays, à partir de la sélection thématique et qualitative éditée par VLB, Suzanne Aubry composa la trame du spectacle au titre éloquent: J'te l'parle mieux quand j'te l'écris.

C'est tout le problème des rapports entre le privé et le public, que l'auteur explique avec Lacoursière dans ces termes:

Ce qu'un personnage historique ne peut confier dans une lettre publique, il va possiblement l'écrire dans une lettre plus confidentielle. Le jeu des significations devient alors fascinant (...) Les lettres peuvent, à cet égard, transformer complètement l'histoire. La correspondance officielle, version "squelettique et musée de cire de l'histoire" alliée à la correspondance privée (la gamme des sentiments, le temps qu'il fait, le quotidien), permettront à l'historien de nuancer et d'approfondir les événements, de les humaniser et même, de leur conférer une précision remarquable ${ }^{5}$.

J'te l'parle mieux... traite surtout de la petite correspondance rurale et citadine des gens ordinaires, souvent pleins d'humour à l'égard des événements qui les assaillent (guerre, crise économique...), parfois désespérés, toujours vifs et d'une authenticité que ne ternit mème pas un certain "rewritting» que l'on devine ça et là. L'apport du théâtre réside surtout dans le surcroît de vitalité que confère la représentation au texte écrit. Cela s'effectue grâce au subterfuge assez simple, ma foi, de la découverte chez quelque brocateur d'un trésor de vieilles lettres et de non moins antiques fripes, par une équipe de joyeux drilles en vacances. C'est «pour le fun» qu'on essaie d'abord les vêtements (habile intégration du costume dans le jeu et du contemporain dans le révolu): robes à dentelles, coiffes, canotiers et chemises à plastron. Par curiosité, ensuite, qu'on se met à lire à haute voix les missives, d'abord ironiquement, puis avec un intérêt de plus en plus soutenu, chacun s'ancrant à mesure dans son rôle. Par jeu, enfin, que s'établit le jeu théâtral, toujours distancié par les changements de costume à vue, sous l'œil ragallardi du brocanteur (Yvon Leroux).

Si la pièce manque parfois de rythme et d'allant, n'évitant pas toujours dans tel passage dramatique l'effet mélo, elle ravit le plus souvent l'amateur de théâtre par l'alternance de telling et de showing qu'appelle ce système de représentation; exemple de la scène de l'amoureux transi qui jette à mesure les brouillons de sa lettre, en allant de l'expression la plus simple et la mieux adaptée à la plus incongrue et tarabiscotée ( "bien écrire", c'est reproduire des figures de style... et des clichés). La mise en scène de l'énonciation ravive chacun des énoncés, jusqu'à la séquence de l'écrivain public qui rédigera pour une bière la lettre exaltée du pauvre colon perdu dans quelque Klondike. Autant de petites scènes croquées sur le vif, dont le spectacle renvoie immanquablement à la lecture, dans un va-et-vient incessant qui donne tout son charme et son efficacité, à travers le jeu des comédiens, à la collaboration Germain-VLB.

Peu d'espace pour parler encore de I'Eskabel dont les dernières produc- 
tions ont pu décevoir l'amateur inconditionnel que j'étais (et que je voudrais bien rester). Noces, bien sûr (février-mars 81), dont les tableaux, toujours soignés et déroutants gardaient le goût baroque des spectacles antérieurs, mais en manquant parfois de cohérence, de "suivi". Chacun d'eux s'affichait un peu comme une pièce en soi, hors-d'œuvre au sens culinaire et hors d'une ceuvre d'ensemble (cas limite du dernier tableau: splendeur hiératique de la progression des comédien(ne)s, en toge fendue, vers les degrés du fond de scène, entre voilages et miroirs réfléchis). Mais hors de toute œuvre concertée. chaque acteur donnant libre cours à son tempérament, ses pulsions, à son trip? L'effet se trouvait malheureusement accusé dans le spectacle suivant, Cristal (mai-juin 81), où le discours féministe tournait à vide dans un volume scénique mal habité par les comédiennes, ou hanté par un appareil scénographique plus ou moins gratuit, en tout cas peu sémantisé par le texte (tubulures métalliques, balançoire, lit ouvert, ombres chinoises tronquées/truquées, etc. $)^{6}$.

Encore moins d'espace, décidément, pour dire Les Pommiers en fleurs. de Serge Sirois, qui reste à mon sens le meilleur texte dramatique publié cette année-là, et le spectacle ayant peut-être eu le plus de succès, à juste titre. Mais plutôt que de risquer une formule à l'emporte-pièce, qui ne rendrait pas justice à la complexité de ce spectacle dérangeant sur l'homosexualité masculine et plus largement sur les problèmes contemporains de la tendresse et de la violence, je me réserve un autre temps, un autre espace pour y revenir...

1. Galilée, dont Martine Dumont a rendu compte dans Spirale 14.

2. J'ai beaucoup changé... dont j'ai rendu compte dans Spirale 13.

3. Moman, de Louisette Dussault, Montréal, Boréal Express, 1981, préface de Lorraine Hébert et al. Auparavant était paru le dossier de Jeu 17 consacré à Dussault, par Lorraine Hébert, Yves Lacroix et moi-même.

4. Chers nous autres, un siècle de correspondance québécoise, par Robert Blondin, en collaboration avec Gilles Lamontagne, chez VLB, Montréal, 1978, 2 tomes.

5. Cf. Suzanne Aubry: «Les lettres, I'histoire et les culottes des Bermudes", in Le Pays théâtral, IV,4; revue du Théâtre d'aujourd'hui.

6. Cela correspond-il au départ ou à la retraite provisoire de Jacques Crête, parallèlement à l'essor de la troupe Opéra-Fête qui, en montant Requiem, se proposait de renouer avec l'esprit eskabélien (mais lequel?). Pour plus de précision sur la scission Eskabel-Opéra-Fête, vair le dossier Eskabel dans Jeu 14 (p. 43-78) et surtout la mise au point de Pierre A. Larocque dans Jeu 16 (p. 231-232).

\section{ERRATUM}

Une erreur apparaît à la note de la p. 204 de Voix et Images, VI, 2 (hiver 1981). II faut y lire une référence à la bibliographie plus détaillée de Jeu 13, sur Jean-Claude Germain. A noter que notre bibliographie, établie antérieurement à celle de Jeu, ne couvrait pas dans le détail le $n^{\circ} 13$ de Jeu, mais y faisait explicitement référence (p. 187). Pour en finir (façon de parler) avec Jeu, on trouvera plus loin dans notre présent numéro, les recensements bibliographiques à jour de cette excellente revue de théâtre. 